pouvoir vous assurer que les hésitations de la rédaction traduisent plus que de la hâte et de l'inachevé ; elles reproduisent des hésitations de la pensée, le sentiment qui poussait d'un rôle de la circulation en géographie humaine bien plus important qu'on n'avait eu l'habitude de le dire. Vers cette époque, dans ses Cours, Demangeon avait commencé de mettre la circulation en tête de sa Géographie économique - avant l'agriculture et l'industrie. Le plan classique en était révolutionné. La mort ne lui laissa pas le temps de donner une forme définitive à l'ordre nouveau dont il sentait le besoin. Le "désarroi "du plan constitue donc une indication d'évolution, une étape dans la méthode... J'en ai discuté plusieurs fois avec lui - et j'ai pensé que ce petit " témoignage " pourrait vous intéresser.

Il intéressera non seulement ma vieille et fidèle amitié pour Demangeon - mais tous les lecteurs de son livre posthume. L'interprétation de Jean Gottmann rend compte, en effet, de ce qu'il y avait d'étrange dans l'espèce de "tournoiement " d'un homme qui, dans tous ses livres et dans tous ses articles, a toujours donnédes modèles de plans lucides, simples et d'une excellence classique. Je dirai volontiers que la lecture de la lettre dont je tiens à donner un extrait à nos lecteurs m'a soulagé d'un poids. J'étais troublé. Comme on l'est toujours quand on ne comprend pas bien. Je ne le suis plus. Et je ne puis que reconnaître Demangeon, "notre Demangeon ", dans ce souci, à la fin de sa vie, de se renouveler encore et toujours, de repenser ses idées et de chercher à serrer do plus près en plus près la réalité. C'est d'un bel exemple - et c'était bien de lui. - L. F.

\title{
MEA CULPA
}

Un lecteur neuchatelois, M. Mermod, m'écrit pour me signaler que, dans les brèves notes que j'ai mises à la suite de Métier d'historien, je signale que " pas une seule fois sauf erreur, dans tout le livre, le mot d'éyolution n'est prononcé ». Et mon correspondant de me demander " pourquoi j'ai l'air de reculer devant ce mot qui lui paraît, en de telles matières, simplement synonyme de développement ". - Après quoi il me fait observer que je fais erreur matériellement, car il relève (notamment p. 9, 73, 92, 94) dans l'ouvrage de Marc Bloch le mot que je pensais absent.

Il a raison. Comment ai-je commis cette erreur ? Simplement parce que j'ai rédigé ma note sur le manuscrit, ou plutôt d'après le manuscrit - et chacun sait qu'on ne tient jamais un manuscrit sous les yeux (quand on n'en est pas soi-même l'auteur) comme on tient un imprimé. - Je m'excuse simplement d'avoir parlé trop vite. Mal défendu par un "sauf erreur » derrière lequel je ne vais pas m'abriter.

Quant à la question de fond? Ce n'est pas moi qui recule devant le mot "évolution ". Si j'ai, après une lecture trop rapide sur ce point, rédigé la note qu'on sait, c'est en me référant à d'anciennes conversations avec Marc Bloch - qui ne tenait pas "évolution" pour synonyme, simplement, de "développement". Et qui était soucieux de bannir de l'histoire (comme moi-même) toutes ces grandes machines, toutes ces "forces " nées de notro cerveau et dont nous faisons sans plus de cérémonie des réalités causales. - L. F. 